\section{Objetivos de Desenvolvimento do Milênio: impacto de ações assistenciais e mudanças socioeconômicas e sanitárias na mortalidade de crianças}

\author{
Millennium Development Goals: the impact \\ of healthcare interventions and changes in \\ socioeconomic factors and sanitation on \\ under-five mortality in Brazil
}

\section{Objetivos de Desarrollo del Milenio: impacto de acciones asistenciales y cambios socioeconómicos y sanitarios en la mortalidad de niños}

\author{
Cristiane da Silva Ramos Marinho 1 \\ Taiana Brito Menezes Flor 1 \\ Josilene Maria Ferreira Pinheiro 1 \\ Maria Ângela Fernandes Ferreira 1
}

doi: 10.1590/0102-311X00191219

\section{Resumo}

No ano 2000, foram estabelecidos os Objetivos de Desenvolvimento do Milênio (ODM), que tiveram como quarta meta a redução da mortalidade na infância (em menores de 5 anos). Desde 2010, o Brasil alcançou a meta proposta. O objetivo deste estudo foi analisar o comportamento da mortalidade na infância e a correlação com os indicadores assistenciais, socioeconômicos e sanitários das regiões brasileiras que contribuíram para o país atingir os ODM. Trata-se de um estudo ecológico, com o uso de dados secundários do Brasil, por Regiões Intermediárias de Articulação Urbana (RIAU), no período de 2001 a 2017. Foram realizadas análises de tendência por meio do joinpoint e modelos de regressão linear múltipla. Constatou-se uma tendência de redução da taxa de mortalidade em menores de 5 anos nos periodos estudados, com maior inflexão estatisticamente significativa entre os anos de 2001 a 2010 $(A A P C=-3,95$; IC95\%: -4,3; -3,6), com menores valores de 2011 a 2015 $(A A P C=-2,35$; IC95\%: -3,7; - 1,0) e estabilização em 2016 e 2017 (AAPC = $-0,07 ;$ ICC $=-4,2 ;+4,3)$. A baixa renda (extrema pobreza) das famílias das crianças e a ausência de escolaridade feminina foram as variáveis que mais se correlacionaram com a taxa da mortalidade na infância $(r=0,649$, $p<0,001$ e $r=0,640, p<0,001$, respectivamente). O cumprimento da quarta meta dos ODM pelo Brasil fez com que o país alcançasse um evidente progresso na redução da taxa de mortalidade na infância, porém percebe-se uma possibilidade de estabilização nesta taxa nos últimos anos. Por outro lado, indicadores sociais e ações assistenciais de saúde foram de grande importância nessa redução, constituindo um desafio ao país a manutenção e evolução das políticas públicas.

Disparidades nos Níveis de Saúde; Saúde da Criança; Mortalidade
Correspondência

C. S. R. Marinho

Universidade Federal do Rio Grande do Norte.

Av. Senador Salgado Filho 1787, Natal, RN 59056-000, Brasil. cristiane_ramos@hotmail.com

1 Universidade Federal do Rio Grande do Norte, Natal, Brasil. 


\section{Introdução}

Os Objetivos de Desenvolvimento do Milênio (ODM) foram propostos no ano 2000 pela Assembleia Geral das Nações Unidas, junto aos 191 países signatários, como um guia para reduzir a pobreza extrema até o ano de 2015. Dentre os oito objetivos propostos, o quarto refere-se à redução em $2 / 3$ das taxas de mortalidade na infância (em menores de 5 anos) em relação ao nível de 1990 1. Isso porque o início da vida concentra o maior número de mortes entre os menores de 5 anos, desencadeando uma redução da sobrevida nas idades mais precoces 2 .

De acordo com dados da Organização das Nações Unidas (ONU), o primeiro dia, semana e mês de vida das crianças são os mais críticos para a sua sobrevivência. Em 2015, dos 5,9 milhões de crianças que morreram antes de completarem 5 anos, aproximadamente 1 milhão foi no dia em que nasceu, outro 1 milhão na primeira semana de vida e cerca de 2,8 milhões durante os primeiros 28 dias 3 . Quanto maior a ocorrência de óbito nos seis primeiros dias de vida, mais complexa é a atuação sobre as causas das mortes, destacando-se a importância do desenvolvimento de ações de saúde e dos serviços de saúde na assistência ao pré-natal, ao parto e ao puerpério 1 .

A mortalidade infantil (em menores de 1 ano) e na infância vem sendo considerada indicadores de grande relevância das condições de vida e saúde de um país, devido à vulnerabilidade a determinantes sociais, de saúde e econômicos 4 . Dentre esses, incluem-se as condições ambientais, fatores demográficos, condições socioeconômicas, o estado nutricional e os fatores relacionados à própria assistência, os quais contribuíram na transição epidemiológica e consequentemente no perfil das principais causas, bem como na evitabilidade das mesmas 5,6.

Diante disso, houve um esforço de âmbito mundial em busca do alcance dos ODM. No início dos anos 1990, o cenário mundial registrava 90 mortes na infância para cada mil nascidos vivos. Por outro lado, em 2015, o mundo conseguiu alcançar 43 mortes por mil nascidos vivos , uma redução de 52,2\% na mortalidade em menores de 5 anos 3 . No Brasil, esses indicadores atingiram a meta proposta no ano de 2010. Em 1990, a taxa de mortalidade na infância era de 59,6 por mil nascidos vivos, portanto, dois terços deste valor representam uma redução de 39,7 por mil nascidos vivos, o que equivale a uma taxa de 19,9 por mil nascidos vivos. A taxa de mortalidade na infância revisada para 2010 foi de 19,4 por mil nascidos vivos, abaixo desse patamar ${ }^{7}$. No entanto, os níveis ainda continuam elevados, face aos grandes desafios que o país enfrenta nessa área, que vão desde disparidades entre regiões e grupos sociais até a precariedade da atenção à mãe e ao recém-nascido.

No ano de 2015, a taxa de mortalidade na infância no Brasil foi de 15,6 por mil nascidos vivos. Esse valor mostrou uma expressiva queda devido, principalmente, ao declínio da mortalidade no primeiro ano de vida, reduzindo de 47,1 por mil nascidos vivos, em 1990, para 13,5 por mil nascidos vivos, em 2015. Nesse mesmo ano, as mortes no componente neonatal ( 0 a 27 dias) corresponderam a $70 \%$ dos óbitos infantis, dos quais $54 \%$ deles ocorreram na primeira semana de vida 8 .

Tais resultados foram influenciados pela assistência à saúde oferecida pelo Sistema Único de Saúde (SUS), que vem conseguindo estreitar as desigualdades de saúde, com melhorias na cobertura e no acesso aos serviços de saúde em todo o país. O SUS oferta cuidados de saúde universais e abrangentes para toda a população brasileira, de forma gratuita, integral, descentralizada e com a ajuda da participação social 9 .

Ademais, a ONU e a Organização Mundial da Saúde (OMS) apontam a necessidade de uma combinação de estratégias juntamente com o próprio SUS, sustentada por políticas, programas e ações, em diferentes áreas, e com maior acesso e cobertura assistencial na melhoria desses indicadores 3. Destacam-se a criação do Estatuto da Criança e do Adolescente (ECA); o Programa Bolsa Família; a Estratégia Saúde da Família (ESF); o Programa Mais Médicos; a Política Nacional de Humanização no Parto e Nascimento; a Rede Cegonha, com diretrizes assistenciais para o parto, nascimento, crescimento e desenvolvimento; e a Política Nacional de Atenção Integral à Saúde da Criança (PNAISC) 10. Além disso, há alguns anos a literatura vem apontando de forma positiva o emprego de políticas públicas voltadas para a melhoria no acesso ao pré-natal, ao abastecimento de água e saneamento como estratégias no combate à mortalidade infantil e na infância 11.

Portanto, há necessidade de manutenção dos progressos alcançados na redução da taxa da mortalidade na infância, assim como do empenho na concretização do compromisso firmado na luta global contra a mortalidade infantil, por meio dos Objetivos de Desenvolvimento Sustentável (ODS), 
que visam a reduzir a mortalidade neonatal para pelo menos 12 por mil nascidos vivos e a mortalidade de crianças menores de 5 anos para pelo menos 25 por mil nascidos vivos, até 2030, no mundo. Além disso, ainda falta um esclarecimento sobre que ações de fato têm maior impacto na redução da mortalidade na infância. Esse conhecimento se torna substancialmente importante neste momento, tendo em vista que há indícios da piora de alguns indicadores a partir do ano de 2016, bem como o ressurgimento de doenças tidas como erradicadas.

Assim sendo, o objetivo deste estudo é analisar a tendência das taxas de mortalidade na infância no Brasil no período de 2001 a 2017 e a correlação com os indicadores assistenciais, socioeconômicos e sanitários das regiões brasileiras que contribuíram para o país atingir os ODM.

\section{Métodos}

Trata-se de um estudo ecológico cuja unidade de análise constituiu as Regiões Intermediárias de Articulação Urbana (RIAU), propostas pelo Instituto Brasileiro de Geografia e Estatística (IBGE). As RIAU são formadas por um grupo de municípios e uma cidade polo, que exerce influência em termos macrorregionais e se caracteriza por apresentar uma articulação interna baseando-se no oferecimento e busca de bens e serviços de alta complexidade. Com base nesses critérios, o Brasil foi dividido em três distintos modelos de regionalização, as Regiões Ampliadas de Articulação Urbana, compostas por 14 territórios; as RIAU, com 161 e as Regiões Imediatas de Articulação Urbana, com 48212.

Para a análise do fenômeno em estudo foi feita a opção pelas RIAU, tendo em vista a complexidade e desigualdade da distribuição populacional no território brasileiro. Nesse sentido, para estabilizar os dados e evitar números aberrantes, os indicadores foram agregados para as 161 regiões.

A coleta de dados abrangeu todos os municípios brasileiros. Os dados para a composição do indicador taxa de mortalidade na infância foram coletados no período de 2001 a 2017 (variável desfecho). Para a assistência à saúde (consulta pré-natal, atendimentos de puericultura, consulta médica em menores de 1 ano, consulta médica em crianças de 1 a 4 anos, visita de enfermagem, visita médica) e condições sanitárias (proporção de domicílios com água da rede geral, proporção de domicílios com dejetos - fezes e urina - a céu aberto, proporção de domicílios com dejetos - fezes e urina - em rede de esgoto), foram coletados os dados nos períodos de 2001 a 2005 e 2011 a 2015. Quanto à escolaridade (proporção de mulheres maiores de 15 anos não alfabetizadas) e à renda (percentual de crianças com baixa renda), os dados coletados referem-se aos anos de 2000 e 2010.

Todos os dados foram obtidos no portal do Departamento de Informática do SUS (DATASUS) e geraram variáveis quantitativas contínuas, conforme especificado no Quadro 1. A coleta e análise dos dados ocorreram nos meses de abril a julho de 2019. Para a construção dos indicadores foi realizada uma média ponderada dos dados municipais de cada RIAU. Essa estratégia foi usada na tentativa de reduzir as discrepâncias que poderiam surgir nesses novos indicadores, caso cidades com portes populacionais diferentes tivessem o mesmo peso na determinação do indicador final.

Com o propósito de avaliar a tendência da mortalidade na infância no Brasil no período de 2001 a 2017, foi utilizado o programa estatístico Joinpoint, versão 4.6.0.0 (http://surveillance.cancer.gov/ joinpoint/), que realiza as estimativas da variação percentual anual (annual percentage change - APC) de uma regressão linear segmentada (jointpont regression) identificando os possíveis pontos de inflexão. As alterações no incremento ou no declínio das taxas de mortalidade são refletidas com base em cada ponto de inflexão 13 . Os testes de significância baseiam-se no método de permutação de Monte Carlo, considerando $\mathrm{p}<0,05$ e no cálculo da variação percentual anual da taxa, utilizando o logaritmo da taxa 14 .

Para a análise da correlação entre a taxa de mortalidade na infância e as variáveis socioeconômicas, sanitárias e relativas à assistência, foi usado o teste de correlação de Pearson e propostos modelos de regressão linear múltipla. Para essas análises, foram usados os períodos de 2001 a 2005 e 2011 a 2015, tanto para a variável desfecho como para as condições sanitárias e assistência à saúde. Por serem oriundas do Censo Demográfico, as variáveis relativas à escolaridade e renda utilizadas foram as dos anos 2000 e 2010. Nessa lógica, optou-se por excluir o período de 2006 a 2010, uma vez que não existiam dados censitários para as referidas variáveis e por elas influenciarem consideravelmente a construção dos modelos finais. A correlação de Pearson permitiu identificar as existências e 


\section{Quadro 1}

Cálculo de indicadores de mortalidade na infância, assistência à saúde, condições socioeconômicas e sanitárias. Regiões Intermediárias de Articulação Urbana (RIAU) brasileiras, 2001 a 2015.

\begin{tabular}{|c|c|c|c|}
\hline & Indicador & Cálculo & Fonte de dados \\
\hline Mortalidade & $\begin{array}{c}\text { Taxa de mortalidade na } \\
\text { infância }\end{array}$ & $\begin{array}{l}\text { Soma do número de óbitos de } \\
\text { crianças menores de } 5 \text { anos } \\
\text { completos residentes na RIAU para } \\
\text { o período/soma do número de } \\
\text { nascidos vivos residentes na RIAU } \\
\text { para o mesmo período x } 1.000\end{array}$ & $\begin{array}{c}\text { DATASUS } \rightarrow \text { Tabnet } \rightarrow \text { Estatísticas Vitais } \rightarrow \text { Mortalidade }-1996 \text { a } \\
\text { 2017, pela CID-10 } \rightarrow \text { Mortalidade Geral } \rightarrow \text { Óbitos por Residência } \\
\text { ( http://tabnet.datasus.gov.br/cgi/deftohtm.exe?sim/cnv/obt10br. } \\
\text { def, acessado em 05/Abr/2019) } \\
\text { DATASUS } \rightarrow \text { Tabnet } \rightarrow \text { Estatísticas Vitais } \rightarrow \text { Nascidos Vivos - } 1994 \\
\text { a } 2017 \rightarrow \text { Nascidos Vivos } \rightarrow \text { Nascimentos por Residência da Mãe } \\
\text { (http://tabnet.datasus.gov.br/cgi/deftohtm.exe?sinasc/cnv/nvbr.def, } \\
\text { acessado em 05/Abr/2019) }\end{array}$ \\
\hline \multirow[t]{5}{*}{$\begin{array}{l}\text { Ações de } \\
\text { saúde }\end{array}$} & Consulta pré-natal & $\begin{array}{l}\text { Soma do número de consultas } \\
\text { pré-natal ocorridas na RIAU para } \\
\text { o período/soma do número de } \\
\text { gestantes cadastradas na RIAU para } \\
\text { o mesmo período }\end{array}$ & $\begin{array}{c}\text { DATASUS } \rightarrow \text { Tabnet } \rightarrow \text { Assistência à Saúde } \rightarrow \text { Atenção Básica } \rightarrow \\
\text { Saúde da Família } \rightarrow \text { De } 1998 \text { a } 2015 \rightarrow \text { Produção e Marcadores } \rightarrow \\
\text { Atendimento Pré-Natal (http://tabnet.datasus.gov.br/cgi/deftohtm. } \\
\qquad \text { exe?siab/cnv/SIABPbr.def, acessado em 02/Mai/2019) } \\
\text { DATASUS } \rightarrow \text { Tabnet } \rightarrow \text { Assistênia à Saúde } \rightarrow \text { Atenção Básica } \\
\rightarrow \text { Saúde da Família - de } 1998 \text { a } 2015 \rightarrow \text { Situação de Saúde } \rightarrow \\
\text { Nºstantes (http://tabnet.datasus.gov.br/cgi/deftohtm.exe?siab/ } \\
\qquad \text { cnv/SIABSbr.def, acessado em 02/Mai/2019) }\end{array}$ \\
\hline & $\begin{array}{l}\text { Atendimentos de } \\
\text { puericultura }\end{array}$ & $\begin{array}{l}\text { Soma do número de atendimentos } \\
\text { de puericultura (consultas médicas e } \\
\text { de enfermagem) realizadas na RIAU } \\
\text { para o período/soma do número } \\
\text { de crianças menores de } 5 \text { anos } \\
\text { cadastradas na RIAU para o mesmo } \\
\text { período }\end{array}$ & $\begin{array}{c}\text { DATASUS } \rightarrow \text { Tabnet } \rightarrow \text { Assistência à Saúde } \rightarrow \text { Atenção Básica } \rightarrow \\
\text { Saúde da Família - de } 1998 \text { a } 2015 \rightarrow \text { Produção e Marcadores } \rightarrow \\
\text { Atendimentos de Puericultura (http://tabnet.datasus.gov.br/cgi/ } \\
\text { deftohtm.exe?siab/cnv/SIABPbr.def, acessado em 02/Mai/2019) } \\
\text { DATASUS } \rightarrow \text { Tabnet } \rightarrow \text { Assistência à Saúde } \rightarrow \text { Atenção Básica } \rightarrow \\
\text { Saúde da Família - de } 1998 \text { a } 2015 \rightarrow \text { Cadastramento Familiar } \rightarrow \\
\text { Mulheres <1ano/Homens<1ano/Mulheres 1a4a/Homens 1a4a } \\
\text { (http://tabnet.datasus.gov.br/cgi/deftohtm.exe?siab/cnv/SIABFbr.def, } \\
\text { acessado em 08/Jun/2019) }\end{array}$ \\
\hline & $\begin{array}{l}\text { Consulta médica em } \\
\text { menores de } 1 \text { ano }\end{array}$ & $\begin{array}{l}\text { Soma do número de consultas } \\
\text { médicas em crianças menores } \\
\text { de } 1 \text { ano ocorridas na RIAU para } \\
\text { o período/soma do número } \\
\text { de crianças menores de } 1 \text { ano } \\
\text { cadastradas na RIAU para o mesmo } \\
\text { período }\end{array}$ & $\begin{array}{l}\text { DATASUS } \rightarrow \text { Tabnet } \rightarrow \text { Assistênia à Saúde } \rightarrow \text { Atenção Básica } \rightarrow \\
\text { Saúde da Família - de } 1998 \text { a } 2015 \rightarrow \text { Produção e Marcadores } \rightarrow \\
\text { Cons.<1 ano (http://tabnet.datasus.gov.br/cgi/deftohtm.exe?siab/ } \\
\text { cnv/SIABPbr.def, acessado em 02/Mai/2019) } \\
\text { DATASUS } \rightarrow \text { Tabnet } \rightarrow \text { Assistência à Saúde } \rightarrow \text { Atenção Básica } \rightarrow \\
\text { Saúde da Família - de } 1998 \text { a } 2015 \rightarrow \text { Cadastramento Familiar } \rightarrow \\
\text { Mulheres <1ano/Homens<1ano (http://tabnet.datasus.gov.br/cgi/ } \\
\text { deftohtm.exe?siab/cnv/SIABFbr.def, acessado em 08/Jun/2019) }\end{array}$ \\
\hline & $\begin{array}{l}\text { Consulta médica em } \\
\text { crianças de } 1 \text { a } 4 \text { anos }\end{array}$ & $\begin{array}{l}\text { Soma do número de consultas } \\
\text { médicas em crianças de } 1 \text { a } 4 \text { anos } \\
\text { ocorridas na RIAU para o período/ } \\
\text { soma do número de crianças de } 1 \text { a } \\
4 \text { anos cadastradas na RIAU para o } \\
\text { mesmo período }\end{array}$ & $\begin{array}{c}\text { DATASUS } \rightarrow \text { Tabnet } \rightarrow \text { Assistência à Saúde } \rightarrow \text { Atenção Básica } \rightarrow \\
\text { Saúde da Família }- \text { de } 1998 \text { a } 2015 \rightarrow \text { Produção e Marcadores } \rightarrow \\
\text { Cons.1a4anos (http://tabnet.datasus.gov.br/cgi/deftohtm.exe?siab/ } \\
\text { cnv/SIABPbr.def, acessado em 02/Mai/2019) } \\
\text { DATASUS } \rightarrow \text { Tabnet } \rightarrow \text { Assistência à Saúde } \rightarrow \text { Atenção Básica } \rightarrow \text { Saúde } \\
\text { da Família - de } 1998 \text { a } 2015 \rightarrow \text { Cadastramento Familiar } \rightarrow \text { Mulheres } \\
\text { 1a4a/Homens 1a4a (http://tabnet.datasus.gov.br/cgi/deftohtm. } \\
\text { exe?siab/cnv/SIABFbr.def, acessado em 08/Jun/2019) }\end{array}$ \\
\hline & Visita de enfermagem & $\begin{array}{l}\text { Soma do número de visitas } \\
\text { realizadas por enfermeiros na RIAU } \\
\text { para o período/soma do total de } \\
\text { famílias cadastradas pela ABS na } \\
\text { RIAU para o mesmo período }\end{array}$ & $\begin{array}{c}\text { DATASUS } \rightarrow \text { Tabnet } \rightarrow \text { Assistência à Saúde } \rightarrow \text { Atenção Básica } \rightarrow \\
\text { Saúde da Família - de } 1998 \text { a } 2015 \rightarrow \text { Produção e Marcadores } \rightarrow \\
\text { Visita Enferm. (http://tabnet.datasus.gov.br/cgi/deftohtm.exe?siab/ } \\
\qquad \text { cnv/SIABPbr.def, acessado em 02/Mai/2019) } \\
\text { DATASUS } \rightarrow \text { Tabnet } \rightarrow \text { Assistência à Saúde } \rightarrow \text { Atenção Básica } \rightarrow \\
\text { Saúde da Família - de } 1998 \text { a } 2015 \rightarrow \text { Cadastramento Familiar } \rightarrow \text { № } \\
\text { Famílias (http://tabnet.datasus.gov.br/cgi/deftohtm.exe?siab/cnv/ } \\
\text { SIABFbr.def, acessado em 08/Jun/2019) }\end{array}$ \\
\hline
\end{tabular}

(continua) 
Quadro 1 (continuação)

\begin{tabular}{|c|c|c|c|}
\hline & Indicador & Cálculo & Fonte de dados \\
\hline $\begin{array}{l}\text { Ações de } \\
\text { saúde }\end{array}$ & Visita médica & $\begin{array}{l}\text { Soma do número de visitas } \\
\text { realizadas por médicos na RIAU para } \\
\text { o período/soma do total de famílias } \\
\text { cadastradas pela atenção básica } \\
\text { de saúde da RIAU para o mesmo } \\
\text { período }\end{array}$ & $\begin{array}{c}\text { DATASUS } \rightarrow \text { Tabnet } \rightarrow \text { Assistência à Saúde } \rightarrow \text { Atenção Básica } \rightarrow \\
\text { Saúde da Família - de } 1998 \text { a } 2015 \rightarrow \text { Produção e Marcadores } \rightarrow \\
\text { Visita Médico (http://tabnet.datasus.gov.br/cgi/deftohtm.exe?siab/ } \\
\text { cnv/SIABPbr.def, acessado em 02/Mai/2019) } \\
\text { DATASUS } \rightarrow \text { Tabnet } \rightarrow \text { Assistência à Saúde } \rightarrow \text { Atenção Básica } \rightarrow \\
\text { Saúde da Família - de } 1998 \text { a } 2015 \rightarrow \text { Cadastramento Familiar } \rightarrow \\
\text { NoFamílias (http://tabnet.datasus.gov.br/cgi/deftohtm.exe?siab/cnv/ } \\
\text { SIABFbr.def, acessado em 08/Jun/2019) }\end{array}$ \\
\hline \multirow[t]{3}{*}{$\begin{array}{l}\text { Condições } \\
\text { sanitárias }\end{array}$} & $\begin{array}{c}\text { Proporção de } \\
\text { domicílios com água da } \\
\text { rede geral }\end{array}$ & $\begin{array}{l}\text { Soma do total de domicílios servido } \\
\text { de água proveniente de uma rede } \\
\text { geral de abastecimento, no domicílio } \\
\text { ou no peridomicílio da RIAU para o } \\
\text { período/soma do total de famílias } \\
\text { cadastradas pela atenção básica } \\
\text { à saúde da RIAU para o mesmo } \\
\text { período }\end{array}$ & $\begin{array}{c}\text { DATASUS } \rightarrow \text { Tabnet } \rightarrow \text { Assistência à Saúde } \rightarrow \text { Atenção Básica } \rightarrow \\
\text { Saúde da Família - de } 1998 \text { a } 2015 \rightarrow \text { Situação de Saneamento } \rightarrow \text { Ab } \\
\text { água-rede públ } \\
\text { (http://tabnet.datasus.gov.br/cgi/deftohtm.exe?siab/cnv/SIABCbr. } \\
\text { def, acessado em 03/Jul/2019) } \\
\text { DATASUS } \rightarrow \text { Tabnet } \rightarrow \text { Assistência à Saúde } \rightarrow \text { Atenção Básica } \rightarrow \\
\text { Saúde da Família - de } 1998 \text { a } 2015 \rightarrow \text { Cadastramento Familiar } \rightarrow \text { № } \\
\text { Famílias } \\
\text { (http://tabnet.datasus.gov.br/cgi/deftohtm.exe?siab/cnv/SIABFbr.def, } \\
\text { acessado em 08/Jun/2019) }\end{array}$ \\
\hline & $\begin{array}{c}\text { Proporção de } \\
\text { domicílios com dejetos } \\
\text { (fezes e urina) a céu } \\
\text { aberto }\end{array}$ & $\begin{array}{l}\text { Soma do total de domicílios com } \\
\text { dejetos (fezes e urina) a céu aberto } \\
\text { da RIAU para o período/soma do } \\
\text { total de famílias cadastradas pela } \\
\text { atenção básica de saúde da RIAU } \\
\text { para o mesmo período }\end{array}$ & $\begin{array}{c}\text { DATASUS } \rightarrow \text { Tabnet } \rightarrow \text { Assistência à Saúde } \rightarrow \text { Atenção Básica } \rightarrow \\
\text { Saúde da Família - de } 1998 \text { a } 2015 \rightarrow \text { Situação de Saneamento } \\
\rightarrow \text { Fez.Urina-céu ab (http://tabnet.datasus.gov.br/cgi/deftohtm. } \\
\text { exe?siab/cnv/SIABCbr.def, acessado em 03/Jul/2019) } \\
\text { DATASUS } \rightarrow \text { Tabnet } \rightarrow \text { Assistência à Saúde } \rightarrow \text { Atenção Básica } \rightarrow \\
\text { Saúde da Família - de } 1998 \text { a } 2015 \rightarrow \text { Cadastramento Familiar } \rightarrow \\
\text { NoFamílias (http://tabnet.datasus.gov.br/cgi/deftohtm.exe?siab/cnv/ } \\
\text { SIABFbr.def, acessado em 08/Jun/2019) }\end{array}$ \\
\hline & $\begin{array}{l}\text { Proporção domicílios } \\
\text { com dejetos (fezes } \\
\text { e urina) em rede de } \\
\text { esgoto }\end{array}$ & $\begin{array}{l}\text { Soma do total de domicílios com } \\
\text { dejetos em rede de esgoto da RIAU } \\
\text { para o período/soma do total de } \\
\text { famílias cadastradas pela atenção } \\
\text { básica de saúde da RIAU para o } \\
\text { mesmo período }\end{array}$ & $\begin{array}{c}\text { DATASUS } \rightarrow \text { Tabnet } \rightarrow \text { Assistência à Saúde } \rightarrow \text { Atenção Básica } \rightarrow \\
\text { Saúde da Família - de } 1998 \text { a } 2015 \rightarrow \text { Situação de Saneamento } \\
\rightarrow \text { Fez.Urina-esgoto (http://tabnet.datasus.gov.br/cgi/deftohtm. } \\
\text { exe?siab/cnv/SIABCbr.def , acessado em 03/Jul/2019) } \\
\text { DATASUS } \rightarrow \text { Tabnet } \rightarrow \text { Assistência à Saúde } \rightarrow \text { Atenção Básica } \rightarrow \\
\text { Saúde da Família - de } 1998 \text { a } 2015 \rightarrow \text { Cadastramento Familiar } \rightarrow \\
\text { NoFamílias (http://tabnet.datasus.gov.br/cgi/deftohtm.exe?siab/cnv/ } \\
\text { SIABFbr.def, acessado em 08/Jun/2019) }\end{array}$ \\
\hline Escolaridade & $\begin{array}{c}\text { Proporção de mulheres } \\
\text { maiores de } 15 \text { anos } \\
\text { não alfabetizadas }\end{array}$ & $\begin{array}{l}\text { Número de mulheres residentes de } \\
15 \text { anos ou mais não alfabetizadas } \\
\text { (não sabem escrever um bilhete } \\
\text { simples no idioma que conhecem) } \\
\text { das RIAU no ano em estudo/ } \\
\text { população de mulheres residentes } \\
\text { maiores de } 15 \text { anos das RIAU no ano } \\
\text { em estudo }\end{array}$ & $\begin{array}{c}\text { DATASUS } \rightarrow \text { Tabnet } \rightarrow \text { Demográficas e Socioeconômicas } \rightarrow \\
\text { Educação - Censos 1991, } 2000 \text { e } 2010 \rightarrow \text { Taxa de Analfabetismo } \rightarrow \\
\text { População de } 15 \text { anos ou mais (Feminino) (http://tabnet.datasus.gov. } \\
\text { br/cgi/deftohtm.exe?ibge/censo/cnv/alfuf, acessado em 03/Jul/2019) } \\
\text { DATASUS } \rightarrow \text { Tabnet } \rightarrow \text { Demográficas e Socioeconômicas } \rightarrow \\
\text { População Residente } \rightarrow \text { Censos (1980, 1991, } 2000 \text { e 2010), Contagem } \\
\text { (1996) e projeções intercensitárias (1981 a 2012), segundo faixa } \\
\text { etária, sexo e situação de domicílio } \rightarrow \text { População Residente (15 anos } \\
\text { ou mais) (http://tabnet.datasus.gov.br/cgi/deftohtm.exe?ibge/cnv/ } \\
\text { popuf.def, acessado em 03/Jul/2019) }\end{array}$ \\
\hline
\end{tabular}

(continua) 
Quadro 1 (continuação)

\begin{tabular}{|c|c|c|c|}
\hline & Indicador & Cálculo & Fonte de dados \\
\hline Renda & $\begin{array}{c}\text { Percentual de crianças } \\
\text { com baixa renda }\end{array}$ & $\begin{array}{c}\text { Número de crianças menores de } \\
14 \text { anos em domicílio com renda } \\
\text { per capita menor de } 1 / 4 \text { salário } \\
\text { mínimo das RIAU no ano em estudo/ } \\
\text { população residente de menores de } \\
14 \text { anos das RIAU no mesmo } \\
\text { ano x } 100\end{array}$ & $\begin{array}{c}\text { DATASUS } \rightarrow \text { Tabnet } \rightarrow \text { Demográficas e Socioeconômicas } \rightarrow \text { Trabalho } \\
\text { e Renda - Censos 1991, } 2000 \text { e } 2010 \rightarrow \text { Proporção de crianças em } \\
\text { situação domiciliar de baixa renda - \% crianças renda dom < 1/4 } \\
\text { SM (http://tabnet.datasus.gov.br/cgi/deftohtm.exe?ibge/censo/cnv/ } \\
\text { crianpobrbr, acessado em 03/Jul/2019) } \\
\text { DATASUS } \rightarrow \text { Tabnet } \rightarrow \text { Demográficas e Socioeconômicas } \rightarrow \text { Trabalho } \\
\text { e Renda - Censos 1991, } 2000 \text { e } 2010 \rightarrow \text { Crianças (menores de } 14 \\
\text { anos) (http://tabnet.datasus.gov.br/cgi/deftohtm.exe?ibge/censo/ } \\
\text { cnv/crianpobrbr, acessado em 03/Jul/2019) }\end{array}$ \\
\hline
\end{tabular}

DATASUS: Departamento de Informática do SUS

magnitudes das correlações entre a variável dependente (mortalidade na infância) e as variáveis independentes para cada período. A força das correlações foi classificada, sendo: $r<0,30$ - correlação fraca; $0,30<\mathrm{r}<0,70$ - correlação moderada; e $\mathrm{r}>0,70$ - correlação forte 15 .

Por fim, foram propostos modelos de regressão linear múltipla para cada um dos períodos analisados. Após a análise da matriz de correlação, foi verificada a presença de colinearidade entre as variáveis "percentual de crianças em domicílio com 1/4 de salário mínimo" e "proporção de mulheres maiores de 15 anos sem alfabetização”. Por esse motivo, foi feita a opção da introdução da variável "escolaridade das mulheres" no modelo de regressão linear múltipla. Após se proceder a análise múltipla, foram checados os pressupostos: ausência de multicolineariade, normalidade dos resíduos, homocedasticidade dos resíduos e ausência de autocorrelação serial. Para a análise bivariada e para a inclusão no modelo final da análise múltipla foram consideradas as variáveis com $\mathrm{p} \leq 0,05$.

A organização do banco foi realizada com o apoio do aplicativo Tabnet (http://www2.datasus.gov. br/DATASUS/index.php?area=02) e do programa IBM SPSS versão 20 (https://www.ibm.com/), no qual foram feitos a agregação do banco, o cálculo dos indicadores e as análises estatísticas.

\section{Resultados}

Entre os anos 2001 e 2017, ocorreram reduções na taxa de mortalidade na infância que passou de 23,39 óbitos por mil nascidos vivos em 2001 para 14,28 óbitos por mil nascidos vivos em 2015, apesar de discreta elevação em 2017, com 14,41 óbitos por mil nascidos vivos. Dessa forma, o estudo constatou que houve tendência significativa de redução da taxa de mortalidade na infância e existência de correlações moderadas entre este desfecho e as variáveis de assistência à saúde, condições sanitárias, relativas à escolaridade e renda.

A tendência das taxas de mortalidade na infância apresentou dois momentos de declínio (inflexão) estatisticamente significativos, nos anos de 2010 (AAPC =-3,95) e 2015 (AAPC = -2,35), o que representa uma redução média anual no período de 2001 a 2010 de 3,95\% e no período de 2011 a 2015 de 2,35\% (Figura 1).

A análise da correlação entre as taxas de mortalidade na infância e os fatores socioeconômicos (renda e escolaridade) e condições sanitárias (destinação dos dejetos e distribuição de água), apresentaram correlação significativa ( $\mathrm{p} \leq$ 0,05) nos dois períodos (2001 a 2005 e 2011 a 2015). Quanto às ações de saúde, não houve um comportamento uniforme nos dois períodos, já que no primeiro foi observada correlação apenas com a variável atendimento pré-natal ( $\mathrm{p} \leq 0,05)$, e no segundo verificouse correlação com as variáveis "visita de enfermagem", "visita médica", "atendimento de puericultura e "consultas de crianças menores de 1 ano" $(\mathrm{p} \leq 0,05)$ (Tabela 1$)$. 


\section{Figura 1}

Tendência das taxas de mortalidade na infância (em menores de 5 anos) nas Regiões de Intermediárias de Articulação Urbana (RIAU) brasileiras - análise por joinpoint (2001 a 2017).

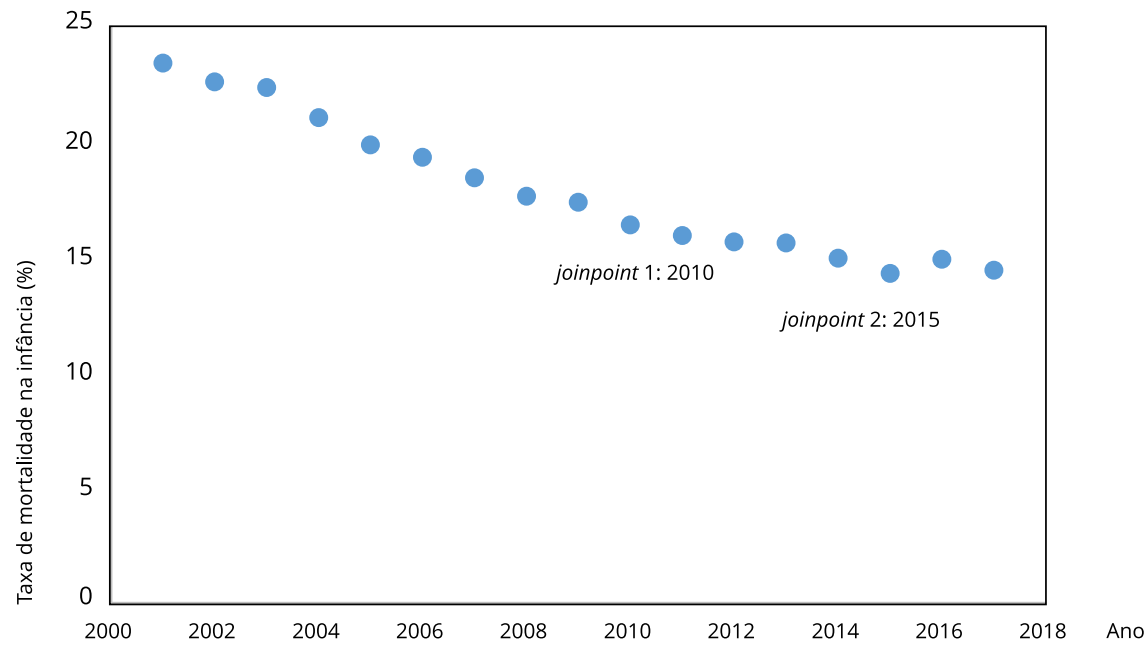

Nota: joinpoint 1: ponto de inflexão do 1o período de 2001 a 2010; joinpoint 2: ponto de inflexão do 2o período de 2011 a 2015 . Média da variação percentual anual (AAPC) significativamente diferentes de zero para alfa $=0,05$ nos períodos de 2001 a 2010 (AAPC = -3,95) e de 2011 a 2015 (AAPC = -2,35) e não significativo no período de 2016 e 2017 (AAPC = -0,07), com intervalo de 95\% de confiança (IC95\%) para todos os períodos.

\section{Tabela 1}

Média, desvio padrão (DP) e correlações de Pearson entre a mortalidade na infância (em menores de 5 anos) e os indicadores socioeconômicos e as ações assistenciais. Regiões Intermediárias de Articulação Urbana (RIAU) brasileiras, 2001 a 2015.

\begin{tabular}{|c|c|c|c|c|c|c|c|c|}
\hline \multirow[t]{2}{*}{ Variáveis } & \multicolumn{4}{|c|}{ Período 1 (2001 a 2005) } & \multicolumn{4}{|c|}{ Período 2 (2011 a 2015) } \\
\hline & Média & DP & $\mathbf{r}$ & Valor de $\mathrm{p}$ & Média & DP & $\mathbf{r}$ & Valor de $p$ \\
\hline Taxa de mortalidade na infância & 22,65 & 5,52 & - & - & 16,04 & 3,35 & - & - \\
\hline Percentual de crianças em domicílio com 1/4 de salário mínimo & 48,46 & 23,60 & 0,649 & $<0,001$ & 31,48 & 19,42 & 0,684 & $<0,001$ \\
\hline Proporção de mulheres maiores de 15 anos sem alfabetização & 0,17 & 0,09 & 0,640 & $<0,001$ & 0,12 & 0,07 & 0,584 & $<0,001$ \\
\hline Proporção de domicílios com dejetos (fezes e urina) a céu aberto & 0,19 & 0,15 & 0,515 & $<0,001$ & 0,06 & 0,06 & 0,534 & $<0,001$ \\
\hline Proporção de domicílios com dejetos (fezes e urina) em rede de esgoto & 0,32 & 0,29 & $-0,364$ & $<0,001$ & 0,20 & 0,15 & $-0,459$ & $<0,001$ \\
\hline Proporção de domicílios servido de água proveniente de uma rede geral & 0,67 & 0,17 & $-0,471$ & $<0,001$ & 0,40 & 0,11 & $-0,303$ & $<0,001$ \\
\hline Proporção de visitas de enfermagem & 0,34 & 0,20 & $-0,043$ & 0,590 & 0,14 & 0,10 & 0,303 & $<0,001$ \\
\hline Proporção de visitas médicas & 0,24 & 0,16 & $-0,061$ & 0,446 & 0,08 & 0,05 & 0,267 & 0,001 \\
\hline Proporção de atendimento pré-natal & 0,86 & 0,49 & 0,156 & 0,049 & 1,16 & 0,46 & 0,147 & 0,063 \\
\hline Proporção de atendimento de puericultura & 1,89 & 1,66 & 0,066 & 0,408 & 2,22 & 2,13 & 0,309 & $<0,001$ \\
\hline Proporção de consultas de crianças menores de 1 ano & 6,32 & 3,76 & $-0,019$ & 0,814 & 6,95 & 5,70 & 0,186 & 0,018 \\
\hline Proporção de consultas de crianças de 1 a 4 anos & 1,27 & 0,73 & $-0,066$ & 0,403 & 1,07 & 0,79 & 0,116 & 0,142 \\
\hline
\end{tabular}


$\mathrm{Na}$ análise de regressão linear múltipla, a variável "proporção de mulheres maiores de 15 anos sem alfabetização" foi determinante na explicação da redução da taxa de mortalidade na infância nos dois períodos estudados $(\mathrm{p}<0,001)$, já o abastecimento de água $(\mathrm{p}=0,009)$ e a realização de consultas em menores de 1 ano $(\mathrm{p}=0,004)$ no primeiro, e os dejetos em rede de esgoto $(\mathrm{p}<0,001)$ no segundo modelo (Tabela 2).

\section{Discussão}

O quarto ODM, que diz respeito à redução de $2 / 3$ da taxa de mortalidade de crianças menores de 5 anos, comparando dados de 1990, foi atingido com êxito pelo Brasil antes do prazo previsto em acordo internacional, que seria no ano de 2015. A superação da meta foi possível devido à combinação de esforços em âmbitos mundial, nacional e local, oportunizando com os resultados o salvamento de milhões de vidas de crianças, bem como a promoção do melhoramento das condições de vida de muitas outras. Diante disso, pode-se perceber que a utilização de intervenções direcionadas, somada ao uso de recursos adequados, estratégias sólidas e vontade política, proporciona até aos países com altos índices de pobreza o desenvolvimento de progressos surpreendentes e sem precedentes 3 .

Durante o período proposto, o Brasil foi um dos 62 países que alcançou o quarto ODM, obtendo uma redução de $73 \%$, acima dos $2 / 3(66,7 \%)$ estabelecidos 16 . Esse resultado chama a atenção para o fato de a sobrevivência infantil, em especial daqueles em situações de vulnerabilidade, continuar a escapar do controle governamental. Isso revela o pequeno progresso mundial, uma vez que dos 191 países que se comprometeram com os ODM, menos de $1 / 3$ conseguiu o alcance da quarta meta e manter o controle da taxa de mortalidade na infância. Doenças de causas evitáveis como pneumonia, diarreia e malária foram responsáveis pela morte de aproximadamente 16 mil crianças menores de 5 anos, diariamente, em todo o mundo, no ano de 2015. Por isso, o declínio drástico na mortalidade evitável na infância é uma das concretizações mais importantes na história da humanidade 3 .

No Brasil, a queda da taxa de mortalidade na infância ocorreu em todas as regiões, a despeito das assimetrias econômicas regionais. Dados do Instituto de Pesquisa Econômica Aplicada (IPEA) apontam que, entre os anos de 1990 e 2011, houve redução na desigualdade regional, com diminuição da taxa de mortalidade na infância em todas as regiões do país, de forma mais acelerada no Nordeste (redução de $76 \%$, média de 6,6\% ao ano) 1 .

Por outro lado, em 2015, face à crise econômica estabelecida no Brasil, percebe-se uma desaceleração acentuada na redução anual da taxa de mortalidade na infância $(0,2 \%)$, quando comparado ao período de 2010 a 2015 (0,8\% ao ano), devido principalmente ao aumento das taxas de pobreza 17. Assim, alerta-se a possibilidade de estabilização das taxas de mortalidade na infância dado os resultados dos últimos dois anos do estudo. Dados recentes evidenciaram aumento de óbitos por causas como coqueluche, ressaltando a importância da manutenção de ações de vigilância, imunização e assistência 18 .

Em 2016, com a justificativa de ajuste das contas públicas, o Governo Federal passou a adotar uma série de medidas de austeridade fiscais, culminando com a Emenda Constitucional no 95 (EC95), que

Tabela 2

Regressão linear multivariada entre a mortalidade na infância (em menores de 5 anos) e indicadores socioeconômicos e ações assistenciais. Regiões Intermediárias de Articulação Urbana (RIAU) brasileiras, 2001 a 2015.

\begin{tabular}{|c|c|c|c|c|c|}
\hline & $r^{2}$ & $\mathbf{r}$ & Variáveis que permaneceram & $\beta$ & Valor de $p$ \\
\hline \multirow[t]{3}{*}{ Período 1 (2001 a 2005) } & 0,462 & 0,679 & Proporção de mulheres não alfabetizadas maiores de 15 anos & 34,747 & $<0,001$ \\
\hline & & & Proporção de domicílios com água da rede geral & $-5,886$ & 0,009 \\
\hline & & & Consulta médica em menores de 1 ano & $-0,255$ & 0,004 \\
\hline \multirow[t]{2}{*}{ Período 2 (2011 a 2015) } & 0,401 & 0,696 & Proporção de mulheres não alfabetizadas maiores de 15 anos & 21,883 & $<0,001$ \\
\hline & & & Proporção de domicílios com dejetos (fezes e urina) em rede de esgoto & $-5,981$ & $<0,001$ \\
\hline
\end{tabular}


dentre outras ações vinculou o gasto público com saúde à inflação até o ano de 2036. Diante disso, projeções apontam para o aumento da mortalidade de crianças como consequência da crise econômica e da subsequente implementação de políticas fiscais de austeridade 9,17 .

A redução no orçamento destinado à saúde provoca efeitos sensíveis na saúde das crianças. Portanto, torna-se imprescindível a adoção de medidas governamentais que visem à promoção da proteção da população, em especial do grupo formado pelas crianças, por meio da manutenção dos investimentos no SUS e em programas sociais, devido à maior vulnerabilidade deste grupo às situações de crise econômica.

No presente estudo, a extrema pobreza ("percentual de crianças em domicílio com 1/4 de salário mínimo") seguida da ausência de escolaridade das mulheres ("proporção de mulheres maiores de 15 anos sem alfabetização") representam as variáveis com as maiores correlações com a mortalidade na infância e, consequentemente, com grande influência na determinação dos modelos finais propostos. Programas de transferência de renda, bem como aqueles que promovem a valorização do salário mínimo ou a distribuição de benefícios sociais, contribuem com a redução das mortes na infância por buscarem favorecer um acesso mais adequado aos serviços de saúde e educação para as famílias, além de condicionar sua permanência 19 . Diante disso, reforça-se a importância de políticas públicas que venham a intervir na renda das famílias e na promoção da educação pública e de qualidade.

Estudos já apontam o potencial impacto desses tipos de programas na pobreza; no estado de saúde e nutricional das famílias, em especial das crianças; no acesso aos alimentos; aos serviços de saúde em diversos países da América Latina, contribuindo consideravelmente para a redução da extrema pobreza e também para a diminuição das desigualdades e melhora nos níveis de saúde 20,21. No ano de 2015, o percentual de pobres no Brasil voltou a crescer, tendo como um de seus fatores contribuintes para este cenário os cortes observados em programas de distribuição de renda, como o Programa Bolsa Família 8. Esse é um programa de transferência condicional de renda, criado pelo Governo Federal no ano de 2003, destinado às famílias em situação de extrema pobreza e àquelas consideradas pobres, com crianças, jovens até 17 anos, gestantes ou lactantes, que recebem um valor monetário a partir do cumprimento de condicionalidades específicas, envolvendo questões relacionadas à saúde, educação e assistência social 22 .

Dados da ONU revelam que as melhorias na sobrevivência infantil ocorreram de forma desigual entre as famílias do Brasil e do mundo. De acordo com inquéritos de agregados familiares, existe uma desproporcionalidade na vulnerabilidade das crianças dos agregados mais pobres quando comparadas com as crianças dos agregados familiares mais ricos, fazendo com que as taxas de mortalidade na infância sejam quase duas vezes superiores nas crianças dos agregados mais pobres do que nas dos mais ricos 3 . Essa desigualdade foi constatada recentemente em um estudo de microssimulação sobre o impacto de medidas de austeridade fiscal no Brasil, com consequente redução de investimentos nos programas de assistência social, como o Programa Bolsa Família. As estimativas apontam para o aumento da morbimortalidade na infância e das desigualdades sociais, com os municípios mais pobres sendo afetados desproporcionalmente 17.

A ausência de escolaridade das mulheres foi outro indicador que se mostrou como forte e consistente preditor para a mortalidade na infância. Nesse sentido, a significativa influência da educação materna sobre a saúde e a sobrevivência de uma criança é uma área importante de investigação 23. A educação materna contribui com a conscientização da mãe sobre boas práticas de cuidados de saúde, nas situações de adoecimento das crianças e disponibilidade de serviços de saúde, o que faz com que seja considerada um fator que pode afetar positivamente a saúde da criança. Um estudo realizado na África subsaariana confirmou que os filhos de mães mais instruídas têm menor risco de morrer antes do quinto ano de vida, em comparação com os filhos de mães sem educação formal 24 . A despeito da constatação de que o grau de escolaridade oportuniza melhores trabalhos e renda, a erradicação do analfabetismo e o investimento na educação precisam vir acompanhados de oportunidades de inserção no mercado de trabalho para possibilitar às mães condições materiais suficientes para os cuidados com os filhos.

Os fatores sanitários também são uma influente e inversa correlação com a taxa de mortalidade na infância. Nesse sentido, as modificações enfrentadas pelo Brasil ao longo dos últimos anos, que culminaram com a melhoria de alguns de seus indicadores socioeconômicos e sanitários, diminuindo 
as dificuldades de acesso e utilização dos meios de promoção, proteção, recuperação e reabilitação da saúde da população, contribuíram de forma direta com a queda da mortalidade na infância 25,26.

Não é novidade que investimentos em saneamento básico, ou seja, na rede de abastecimento de água potável, esgoto sanitário, tratamento de resíduos sólidos, drenagem e limpeza urbana são de extrema relevância na promoção da saúde pública por meio da prevenção de doenças e da melhoria da qualidade de vida e do bem-estar das pessoas. Todavia, as ações públicas de saneamento, implementadas no Brasil, recebem elevada pressão política e econômica, resultando em descontinuidade tanto pelos baixos investimentos quanto pelas fragilidades institucionais e legais 27.

Ao longo dos últimos anos, observamos um aumento na cobertura dos serviços sanitários, porém, a universalização do acesso a este tipo de serviço no Brasil continua sendo um desafio que ainda está longe de ser alcançado. Diversos estudos retratam essa dificuldade, enfatizando a diferença de acesso existente entre as regiões brasileiras, os níveis de renda e a escolaridade, resultando na produção de uma sociedade apartada, com acesso diferenciado a direitos considerados fundamentais, como é o caso do saneamento básico (Lei no 11.445) 28,29.

Em 2010, recebiam atendimento adequado para abastecimento de água, esgotamento sanitário e manejo dos resíduos sólidos, 59,4\%, 39,7\% e 58,6\% da população brasileira, respectivamente, gerando um déficit bastante significativo em todos os componentes do saneamento básico, o que equivale a milhões de pessoas vivendo em ambientes insalubres, suscetíveis a diversos riscos que comprometem a sua saúde 30. Esse contexto de exclusão e desigualdade, acompanhado, em algumas situações, de baixa qualidade dos serviços ofertados é o produto de um modelo de desenvolvimento baseado no modo de produção capitalista, que por sua vez, promove contradições, antagonismo e iniquidades 31 .

Por outro lado, sabe-se da necessidade de implantação de programas de saneamento básico como prioridade, em especial em áreas de maior vulnerabilidade por representarem suporte efetivo na melhoria da saúde, bem como na redução da mortalidade das crianças e da população de uma forma geral 32. Portanto, é primordial o desenvolvimento de políticas públicas que busquem a garantia do acesso universal a esse serviço, com foco nas demandas da população por meio de investimentos adequados, participação social, que não se restrinja à elaboração de projetos, nem se prenda exclusivamente às concepções neoliberais que limitam a atuação do Estado.

A saúde é o produto da interação entre variáveis sociais, ambientais e econômicas que desencadeiam pressões sobre as condições e a qualidade de vida. A fragilidade no sistema público e uma infraestrutura sanitária deficiente impactam de forma direta no desenvolvimento de doenças infecciosas e, consequentemente, na morbimortalidade de crianças, em especial nos países pobres e em desenvolvimento 33 .

Em 2007, foi criado o Programa de Aceleração do Crescimento (PAC), momento em que se observa uma ampliação significativa dos recursos destinados ao saneamento no país. A concepção do PAC envolveu um conjunto de medidas legislativas, administrativas e de políticas de investimento, visando a estimular o crescimento da economia do país, destinando recursos a áreas prioritárias de infraestrutura (logística, energia, transportes, habitação e saneamento, entre outras). Entretanto, no que se refere às ações de saneamento, embora o PAC tenha promovido a ampliação dos recursos, bem como favorecido a retomada dos investimentos na área, não foram suficientes para desenvolver medidas e ações que se aproximassem dos pressupostos das diretrizes da Lei Nacional de Saneamento Básico 29. Por se tratar de um programa que objetivava contribuir com o desenvolvimento do país, colocando-o em um patamar de crescimento econômico diferenciado, o PAC manteve-se fiel a uma lógica econômica e tecnocrática, priorizando a maior parte do recurso orçamentário em setores que favorecessem um ganho de capital, ficando as ações de saneamento em um plano secundário.

Aliadas aos determinantes contextuais, as ações de saúde são de extrema importância na determinação da mortalidade na infância. No presente estudo, a assistência médica oferecida pela equipe de saúde para menores de 1 ano apresentou influência na redução da taxa de mortalidade na infância. Ações que contribuam com o acesso aos serviços de saúde, promovam incentivos quanto ao aumento da cobertura vacinal, da segurança alimentar e nutricional, da ampliação da cobertura da ESF e da melhoria geral das condições de vida possibilitam que muitas causas de óbitos na infância sejam evitadas, de modo que os agravos à saúde jamais ou raramente evoluam para o óbito 34,35.

A implantação do Programa de Agentes Comunitários de Saúde e da ESF, nos anos 1990, viabilizaram a ampliação do acesso aos serviços de atenção básica à saúde ao oportunizar a interiorização 
de equipes de saúde da família, culminando no aumento da cobertura da atenção à saúde reprodutiva e infantil 8. Em 1995, a ESF estava presente em 115 municípios brasileiros e em dezembro de 2013 era realidade em mais de $95 \%$ das cidades brasileiras ${ }^{1}$. A ESF tem melhorado os resultados de saúde e reduzido as desigualdades de saúde no Brasil. Ademais, a consolidação da ESF tem contribuído com a redução das taxas de mortalidade infantil, na infância e materna, com a diminuição das taxas de doenças imunopreveníveis e de internações por condições evitáveis 24. Pesquisa realizada sobre a avaliação do impacto do Programa Saúde da Família na mortalidade infantil no Brasil, 1990-2002, revelou que a cada 10\% de ampliação da cobertura da ESF gerou-se uma redução de 4,6\% da mortalidade infantil 36.

A expansão da cobertura da atenção primária, sustentada pela ESF, foi fundamental no alcance das atuais taxas de mortalidade na infância, no entanto, a manutenção dos resultados alcançados está diretamente relacionada com o financiamento da saúde. Daí a necessidade de manutenção, com consequente ampliação, de recursos orçamentários para o SUS.

Por fim, o estudo mostrou que indicadores relativos à escolaridade, condições sanitárias e ações de saúde exercem determinação na mortalidade na infância, atuando de forma considerada na diminuição da taxa de mortalidade de menores de 5 anos, compondo os modelos finais da análise múltipla empreendida.

\section{Considerações finais}

A análise de tendência da mortalidade na infância mostrou redução significativa na maior parte do período estudado (2001 a 2015), com tendência à estabilização a partir de 2015. Isso levanta um alerta para a vigilância dos fatores envolvidos com esse indicador, especialmente pelo fato do Brasil ter atingido a quarta meta dos ODM e alcançado as menores taxas de mortalidade na infância de sua história. A utilização da metodologia por joinpoint fez com que o artigo possibilitasse uma análise estatística da tendência da mortalidade na infância, destacando os pontos temporais em que se observaram as maiores inflexões, oportunizando o estudo dos fatores envolvidos com os resultados obtidos naquele período.

Foram encontradas correlações entre os fatores econômicos, educacionais, sanitários e de saúde com a melhoria da mortalidade na infância nos períodos estudados. Contudo, percebeu-se grande influência da escolaridade de mulheres e das condições sanitárias na proposição de um modelo de regressão múltipla, sugerindo que estes indicadores contribuem para o acesso e cuidado à saúde.

Como um desafio no contexto dos cortes de gastos públicos, destaca-se a necessidade do fortalecimento do SUS, devido à importância de suas ações de saúde em direção ao alcance das metas quanto à mortalidade na infância proposta pelos ODS.

Enfatiza-se ainda o desempenho do Brasil quanto à superação da quarta meta do ODM, assim como todas as dificuldades para a manutenção dos resultados alcançados, diante de um contexto de crise política e econômica, atrelado à implantação de medidas de austeridade fiscal. Assim, reforçase a necessidade de manutenção das políticas de proteção à vida, bem como de proposição de novas políticas públicas que venham a agir sobre os determinantes sociais da saúde que interferem na morbimortalidade na infância, garantindo a sustentabilidade das ações para os próximos anos. Os resultados obtidos podem oferecer lições sobre políticas a serem seguidas, bem como sobre os caminhos a serem evitados. 


\section{Colaboradores}

C. S. R. Marinho participou da concepção e projeto, a análise e interpretação dos dados, redação do artigo e revisão crítica relevante do conteúdo intelectual. T. B. M. Flor e J. M. F. Pinheiro colaboraram na análise e interpretação dos dados, redação do artigo e revisão crítica relevante do conteúdo intelectual. M. A. F. Ferreira contribuiu na revisão crítica relevante do conteúdo intelectual e na aprovação final da versão a ser publicada.

\section{Informaçãoes adicionais}

ORCID: Cristiane da Silva Ramos Marinho (00000003-3825-3057); Taiana Brito Menezes Flor (0000-0001-5164-8446); Josilene Maria Ferreira Pinheiro (0000-0003-0564-4828); Maria Ângela Fernandes Ferreira (0000-0002-6142-948X).

\section{Agradecimentos}

Ao Programa de Pós-graduação em Saúde Coletiva (PPGSCOL) daUniversidade Federal do Rio Grande do Norte (UFRN) e à Faculdade de Ciências da Saúde do Trairi (FACISA) da UFRN.

\section{Referências}

1. Instituto de Pesquisa Econômica Aplicada. Objetivos de Desenvolvimento do Milênio: relatório nacional de acompanhamento. Brasília: Instituto de Pesquisa Econômica Aplicada; 2014.

2. Kale PL, Silva KS, Saraceni V, Coeli CM, Torres TZG, Vieira FMSB, et al. Ameaça à vida ao nascer: uma análise das causas de morte e estimativa de sobrevida de menores de cinco anos em coortes de nascidos vivos. Cad Saúde Pública 2019; 35:e0186418.

3. Nações Unidas. Relatório sobre os Objetivos de Desenvolvimento do Milênio 2015. Nova York: Nações Unidas; 2015.

4. Departamento de Vigilância de Doenças e Agravos não Transmissíveis e Promoção da Saúde, Secretaria de Vigilância em Saúde, Ministério da Saúde. Saúde Brasil 2017: uma análise da situação de saúde e os desafios para o alcance dos objetivos de desenvolvimento sustentável. Brasília: Ministério da Saúde; 2018.

5. França EB, Lansky S, Rego MAS, Malta DC, França JS, Teixeira R, et al. Principais causas da mortalidade na infância no Brasil, em 1990 e 2015: estimativas do estudo de Carga Global de Doença. Rev Bras Epidemiol 2017; 20 Suppl 1:46-60

6. Teixeira JAM, Araujo WRM, Maranhão AGK, Cortez-Escalante JJ, Rezende LFM, Matijasevich A. Mortality in the first day of life: trends, causes of death and avoidability in eight Brazilian Federative Units, between 2010 and 2015. Epidemiol Serv Saúde 2019; 28:e2018132.

7. Em 2011, esperança de vida ao nascer era de 74,08 anos. Agência IBGE Notícias 2012; 29 nov. https://agenciadenoticias.ibge.gov.br/ agencia-sala-de-imprensa/2013-agencia-denoticias/releases/14321-asi-em-2011-espe ranca-de-vida-ao-nascer-era-de-7408-anos.

8. Leal MC, Szwarcwald CL, Almeida PVB, Aquino EML, Barreto ML, Barros F, et al. Saúde reprodutiva, materna, neonatal e infantil nos 30 anos do Sistema Único de Saúde (SUS). Ciênc Saúde Colet 2018; 23:1915-28.

9. Castro MC, Massuda A, Almeida G, Menezes-Filho NA, Andrade MV, Noronha KVMS, et al. Brazil's Unified Health System: the first 30 years and prospects for the future. Lancet 2019; 394:345-56

10. Departamento de Ações Programáticas Estratégicas, Secretaria de Atenção à Saúde, Ministério da Saúde. Política Nacional de Atenção Integral à Saúde da Criança: orientações para implementação. Brasília: Ministério da Saúde; 2018.

11. Carvalho RAS, Santos VS, Melo CM, Gurgel RQ, Oliveira CCC. Desigualdades em saúde: condições de vida e mortalidade infantil em região do nordeste do Brasil. Rev Saúde Pública $2015 ; 49: 5$ 
12. IBGE divulga divisão urbano-regional do Brasil. Agência IBGE Notícias 2013; 18 jun. https://agenciadenoticias.ibge.gov.br/agenciasala-de-imprensa/2013-agencia-de-noticias/ releases/14421-asi-ibge-divulga-divisao-ur bano-regional-do-brasil.

13. Ferreira DB, Mattos IE. Tendência da mortalidade por câncer de mama em mulheres no Estado do Rio de Janeiro, Brasil, 1996-2011. Ciênc Saúde Colet 2015; 20:895-903.

14. Barbosa IR, Souza DLB, Bernal MM, Costa ICC. Desigualdades regionais na mortalidade por câncer de colo de útero no Brasil: tendências e projeções até o ano 2030. Ciênc Saúde Colet 2016; 21:253-62.

15. Sánchez-Villegas A, Martín-Calvo N, Martínez-González MA. Correlación y regresión lineal simple. In: Martínez-González MA, Sánchez-Villegas A, Atucha ET, Farjado JF, editors. Bioestadística amigable. 3a Ed. Barcelona: Elsevier; 2014. p. 269-326.

16. Batista Filho M, Cruz RSBLC. A saúde das crianças no mundo e no Brasil. Rev Bras Saúde Matern Infant 2015; 15:451-4.

17. Rasella D, Basu S, Hone T, Paes-Sousa R, Ocké -Reis CO, Millett C. Morbidade e mortalidade infantil associadas a respostas políticas alternativas à crise econômica no Brasil: um estudo de micro-simulação nacional. PLoS Med 2018; 15:e1002570.

18. Malta DC, Prado RR, Saltarelli RMF, Monteiro RA, Souza MFM, Almeida MF. Mortes evitáveis na infância, segundo ações do Sistema Único de Saúde, Brasil. Rev Bras Epidemiol 2019; 22:e190014.

19. Associação Brasileira de Saúde Coletiva. Especial Abrasco sobre o aumento da mortalidade infantil e materna no Brasil. Rio de Janeiro: Associação Brasileira de Saúde Coletiva; 2018.

20. Lagarde M, Haines A, Palmer N. The impact of conditional cash transfers on health outcomes and use of health services in low and middle income countries. Cochrane Database Syst Rev 2009; (4):CD008137.

21. Segura-Perez S, Grajeda R, Perez-Escamilla R. Conditional cash transfer programs and the health and nutrition of Latin American children. Rev Panam Salud Pública 2016; 40:12437.

22. Silva ESA, Paes NA. Programa Bolsa Família e a redução da mortalidade infantil nos municípios do semiárido brasileiro. Ciênc Saúde Colet 2019; 24:623-30.

23. Oyekale AS, Maselwa TC. Maternal education, fertility, and child survival in Comoros. Int J Environ Res Public Health; 15:2814.
24. Bado AR, Sathiya Susuman A. Women's education and health inequalities in under-five mortality in selected Sub-Saharan African countries, 1990-2015. PLoS One 2016; 11:e0159186.

25. Victora CG, Aquino EM, Leal MC, Monteiro CA, Barros FC, Szwarcwald CL. Maternal and child health in Brazil: progress and challenges. Lancet 2011; 377:1863-76.

26. Oliveira VH, Medeiros CN. Regime de chuvas e saúde infantil no Estado do Ceará: evidências para os municípios em anos censitários (19912010). Nova Economia 2019; 29:307-38.

27. Coelho C, Borja P, Santos M. Desigualdades de acesso e qualidade dos serviços de saneamento básico da bacia hidrográfica do Rio Camarajipe - Salvador - BA. Bahia Análise \& Dados 2020; 29:153-73.

28. Mendonça MJC, Motta RS. Saúde e Saneamento no Brasil. Planej Polít Públicas 2007; 30:15-30.

29. Cunha MA, Borja PC. O programa de aceleração do crescimento no Estado da Bahia e os desafios da universalização do saneamento básico. Revista Brasileira de Gestão Urbana 2018; 10 Suppl 1:173-85.

30. Secretaria Nacional de Saneamento Ambiental, Ministério das Cidades. Plano Nacional de Saneamento Básico (Plansab). Brasília: Ministério das Cidades; 2013.

31. Borja PC. Política pública de saneamento básico: uma análise da recente experiência brasileira. Saúde Soc 2014; 23:432-47.

32. Rasella D. Impacto do Programa Água para Todos (PAT) sobre a morbi-mortalidade por diarreia em crianças do Estado da Bahia, Brasil. Cad Saúde Pública 2013; 29:40-50.

33. Teixeira JC, Gomes MHR, Souza JA. Associação entre cobertura por serviços de saneamento e indicadores epidemiológicos nos países da América Latina: estudo com dados secundários. Rev Panam Salud Pública 2012; 32:41925.

34. Moreira KFA, Oliveira TS, Gonçalves TA, Moura CO, Maluf SN, Tavares RSA, et al. Child mortality in the last five-year periods in the city of Porto Velho, RO, Brazil. J Hum Growth Dev 2014; 24:86-92.

35. Coordenação de Epidemiologia e Informação, Secretaria Municipal de Saúde de São Paulo. Mortalidade infantil no Município de São Paulo: tendências recentes e desigualdades socioespaciais. Boletim Eletrônico CEInfo 2014; Ano IX, no 8.

36. Macinko J, Guanais FC, Fatima M, Souza M. Evaluation of the impact of the Family Health Program on infant mortality in Brazil, 19902002. J Epidemiol Community Health 2006; 60:13-9. 


\section{Abstract}

The United Nations approved the Millennium Development Goals (MDGs) in 2000, including Target 4.A, or a two-thirds reduction in underfive mortality by 2015. Brazil reached this target in 2010. The current study aimed to analyze the trend in under-five mortality and the correlation with healthcare, socioeconomic, and sanitation indicators in Brazil's major geographic regions that helped the country meet the MDGs. This was an ecological study using secondary data for Brazil according to Intermediate Urban Linkage Regions (RIAU in Portuguese) from 2001 to 2017. Analyses of tendencies were performed with joinpoint and multiple linear regression models. The study showed a downward trend in the under-five mortality rate during the periods studied, with the largest statistically significant change from 2001 to 2010 ( $A A P C=-3.95 ; 95 \% \mathrm{CI}:-4.3 ;-3.6)$, the lowest changes from 2011 to 2015 (AAPC $=-2.35$; 95\% CI: - 3.7; - 1.0), and stabilized rates in 2016 and $2017($ AAPC $=-0.07 ;$ ICC $=-4.2 ;+4.3)$. Low income (extreme poverty) in the children's families and absence of maternal schooling were the variables most closely correlated with under-five mortality rate $(r=0.649, p<0.001$ and $r=0.640, p<$ 0.001 , respectively). The fact that Brazil met the fourth target in the MDGs reflected the country's progress in reducing the under-five mortality rate, but the data suggest the rate's possible stabilization in recent years. Meanwhile, social and healthcare indicators revealed the importance of this reduction, challenging the country to maintain and further improve its public policies in this area.

Health Status Disparities; Child Health; Mortality

\section{Resumen}

En el año 2000 se establecieron los Objetivos de Desarrollo del Milenio (ODM), cuya cuarta meta era la reducción de $2 / 3$ de la mortalidad en la infancia (en menores de 5 años). Desde 2010, Brasil alcanzó la meta propuesta. El objetivo de este estudio fue analizar el comportamiento de la mortalidad en la infancia y la correlación con los indicadores asistenciales, socioeconómicos y sanitarios de las regiones brasileñas que contribuyeron a que país alcanzara los ODM. Se trata de un estudio ecológico, con la utilización de datos secundarios de Brasil, por Regiones Intermediarias de Coordinación Urbana (RIAU en Portugués), durante el período de 2001 a 2017. Se efectuaron análisis de tendencia a través de joinpoint y modelos de regresión lineal múltiple. Se constató una tendencia de reducción de la tasa de mortalidad en menores de 5 años durante los períodos estudiados, con mayor inflexión estadísticamente significativa durante el periodo de 2001 a 2010 (AAPC = -3,95; IC95\%: - 4,3; -3, 6), con menores valores en 2011 a $2015($ AAPC $=-2,35$; IC95\%: - 3, 7; - 1,0) y estabilización en 2016 y $2017($ AAPC $=-0,07 ;$ ICC $=$ $-4,2 ;+4,3)$. La renda baja (extrema pobreza) de las familias de los niños y la ausencia de escolaridad femenina fueron las variables que más se correlacionaron con la tasa de la mortalidad en la infancia $(r=0,649, p<0,001$ y $r=0,640, p<0,001$, respectivamente). El cumplimiento de la cuarta meta de los ODM, por parte de Brasil, consiguió que el país alcanzase un evidente progreso en la reducción de la tasa de mortalidad en la infancia, pese a que se percibe una posibilidad de estabilización en esta tasa en los últimos años. Por otro lado, indicadores sociales y acciones asistenciales de salud se revelaron de gran importancia en esa reducción, al constituirse en un desafío para el país en cuanto al mantenimiento y evolución de las políticas públicas.

Disparidad en el Estado de Salud; Salud del Niño; Mortalidad
Recebido em 30/Set/2019

Versão final reapresentada em 21/Fev/2020

Aprovado em 20/Mar/2020 